\title{
The effect of optical dilatation before retrograde intrarenal surgery on success and complications: results of the RIRSearch group study.
}

Eyup Burak Sancak ${ }^{1}$, Cem Basatac ${ }^{2}$, Murat Akgul ${ }^{3}$, Onder Cinar ${ }^{4}$, Oktay Ozman $^{2}$, Cenk Murat Yazici ${ }^{3}$, and Haluk Akpinar ${ }^{2}$

${ }^{1}$ Canakkale Onsekiz Mart Universitesi

${ }^{2}$ Affiliation not available

${ }^{3}$ Namik Kemal University Faculty of Medicine

${ }^{4}$ Zonguldak Bulent Ecevit University

April 4, 2021

\begin{abstract}
Aims: The guidelines propose optical dilatation before retrograde intrarenal surgery (RIRS), but there are no evidence-based studies concerning the impact of optical dilatation with semirigid ureteroscopy (sURS) in the literature. The aim of this study was to evaluate the effect of optical dilatation through sURS prior to the procedure on the success and complications of RIRS. Methods: In a multicentre retrospective study, 422 patients were included in the study. The patients were divided into two groups according to whether sURS was to be performed. Patients' demographics, stone parameters and operative outcomes were compared. Surgical success was defined as no or up to 3-mm residual stone fragments without the need for additional procedures. The independent predictors for surgical success were determined with a multivariable logistic regression model. Results: Of the 422 patients, $133(31.5 \%)$ were in the sURS group and 289 (68.5\%) were in the non-sURS group. Operation time in the sURS group was significantly long $(\mathrm{p}<0.0001)$. A ureteral access sheath (UAS) could not be placed in four $(3.0 \%)$ patients in the sURS group, nor in $25(8.7 \%)$ patients in the non-sURS group $(\mathrm{p}=0.03)$. Compared with the non-sURS group, the intraoperative complication rate was low in the sURS group $(14(4.8 \%)$ vs $1(0.8 \%), \mathrm{p}=0.04)$. The surgical success rate was higher in the sURS group $(\mathrm{p}=0.002)$. Nevertheless, sURS had no independent effect on surgical success. We have found two independent predictors for surgical success rate: stone number $(\mathrm{p}<0.0001$, OR:2.28) and failed UAS placement ( $\mathrm{p}=0.035$, OR:3.49) Conclusion: Optical dilatation with sURS before RIRS increases surgical success by raising the rate of UAS placement and reducing the rate of intraoperative complications. We suggest that this method can be routinely applied in the group of patients who have not been passively dilated with a JJ stent
\end{abstract}

The effect of optical dilatation before retrograde intrarenal surgery on success and complications: results of the RIRSearch group study.

Eyup Burak Sancak ${ }^{1}$, Cem Basatac ${ }^{2}$, Hacı Murat $\mathrm{Akgul}^{3}$, Onder Cinar ${ }^{4}$, Oktay Ozman ${ }^{5}$, Cenk Murat Yazıci ${ }^{3}$, Haluk Akpinar ${ }^{2}$

${ }^{1}$ Canakkale Onsekiz Mart University Faculty of Medicine, Department of Urology, Canakkale/Turkey

${ }^{2}$ Florence Nightingale Hospital, Department of Urology Istanbul/ Turkey

${ }^{3}$ Tekirdag Namık Kemal Faculty of Medicine, Department of Urology, Tekirdag/ Turkey 


\section{${ }^{4}$ Zonguldak Bulent Ecevit University Faculty of Medicine, Department of Urology, Zonguldak/}

Turkey

${ }^{5}$ Istanbul Gaziosmanpasa Training and Research Hospital, Department of Urology, Istanbul/ Turkey

Short Title: Optical dilatation and intrarenal surgery.

\section{Corresponding author}

Eyup Burak Sancak, Assoc. Prof.

Department of Urology.

Canakkale Onsekiz Mart University Faculty of Medicine.

Canakkale, Kepez, 17100, Turkey

Phone/Fax +90 $5323782204 /+902862180393$

E-maileyupburaksancak@comu.edu.tr

\section{ABSTRACT}

Aim: The guidelines propose optical dilatation before retrograde intrarenal surgery (RIRS), but there are no evidence-based studies concerning the impact of optical dilatation with semirigid ureteroscopy (sURS) in the literature. The aim of this study was to evaluate the effect of optical dilatation through sURS prior to the procedure on the success and complications of RIRS.

Methods: In a multicentre retrospective study, 422 patients were included in the study. The patients were divided into two groups according to whether sURS was to be performed. Patients' demographics, stone parameters and operative outcomes were compared. Surgical success was defined as no or up to 3-mm residual stone fragments without the need for additional procedures. The independent predictors for surgical success were determined with a multivariable logistic regression model.

Results: Of the 422 patients, $133(31.5 \%)$ were in the sURS group and 289 (68.5\%) were in the nonsURS group. Stone characteristics and patients' demographics were similar among the groups. Operation time in the sURS group was significantly long $(p<0.0001)$. A ureteral access sheath (UAS) could not be placed in four (3.0\%) patients in the sURS group, nor in $25(8.7 \%)$ patients in the non-sURS group $(p=0.03)$. Compared with the non-sURS group, the intraoperative complication rate was low in the sURS group (14 (4.8\%) vs $1(0.8 \%), p=0.04)$. The surgical success rate was higher in the sURS group $(p=0.002)$. Nevertheless, sURS had no independent effect on surgical success. We have found two independent predictors for surgical success rate: stone number $(p<0.0001, \mathrm{OR}: 2.28)$ and failed UAS placement $(p=0.035$, OR:3.49)

Conclusions: Optical dilatation with sURS before RIRS increases surgical success by raising the rate of UAS placement and reducing the rate of intraoperative complications. We suggest that this method can be routinely applied in the group of patients who have not been passively dilated with a JJ stent.

Keywords: Flexible ureteroscopy; optical dilatation; retrograde intrarenal surgery, semirigid ureteroscopy, ureteral access sheath.

\section{INTRODUCTION}

Improvements in surgical techniques and endourological devices over recent years have led to significant changes in treatment modalities for kidney stones. Among these, retrograde intrarenal surgery (RIRS) is now considered one of the first-line treatment options, with high stone-free rates and lower morbidity for

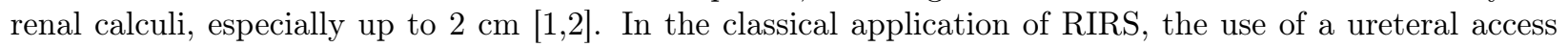
sheath (UAS) may provide significant advantages by decreasing intra-renal pressure, improving visibility, and allowing easy insertion of the endourological equipment into the collecting system [1,3]. However, in some patients, the placement of a UAS is quite difficult. Several strategies are suggested to allow easy 
insertion of a UAS during RIRS. Some authors defend routine stent placement before RIRS to provide passive ureteral dilatation [1,4]. Despite its effectiveness and reliability, this method requires a two-stage procedure. The other option, known as active ureteral dilatation performed by balloon or coaxial dilator before UAS, may cause significant ureteral injury $[1,5]$. On the other hand, for the past ten years EAU guidelines have recommended doing optical dilatation with semirigid ureteroscopy (sURS) before RIRS to facilitate the process $[6,7]$.

Semirigid ureteroscopy provides optical ureteral dilatation with easier ureteral access and inspection of upper urinary tract anatomy for possible pathologies such as stones, strictures, or tumours. In addition, ureteral diameter and compliance can be evaluated to select the proper UAS size [5,8]. However, the effects of optical dilatation on RIRS success and complications have not yet been investigated. In this multicentre study, we aimed to evaluate the effects of optical dilatation through sURS on the surgical success and complication rates of RIRS.

\section{METHODS:}

The present study conducted in accordance with the tenets of the Declaration of Helsinki and with the approval of Ethics Committee of Onsekiz Mart University School of Medicine (Approval number: 26.02.2020/2019-04). A total of 515 patients who had undergone RIRS for renal and upper ureteral stones between February 2016 and January 2020 at four referral centres in Turkey were included in this study. All operations were carried out by senior surgeons with a minimum of five years of experience at these centres. Patients' characteristics, including age, gender, the side and size of the stone, body mass index, previous stone treatment history, and operative outcomes, were entered into each centre's database retrospectively. The patients with incomplete records and/or known renal anatomical abnormalities were excluded from the study. Patients requiring preoperative or intraoperative active or passive ureteral dilatation and undergoing ipsilateral ureteral surgery were also excluded. The remaining 422 patients were included in this study.

All patients underwent a preoperative radiologic evaluation with non-contrast computed tomography (NCCT). The stone characteristics were recorded from NCCT findings. Stone size was determined by the largest diameter of the main stone. The mean Hounsfield unit was calculated using the elliptical region of interest incorporated into the largest stone area in an axial image of NCCT [9].

The patients were divided into two groups based on the use of sURS or not. Patients' demographics and operative outcomes were compared between groups. The intraoperative and postoperative surgical complications were noted. The primary outcome was to define whether optical ureteral dilatation with sURS before the procedure provides high surgical success along with a low complication rate. The secondary outcome was to evaluate independent predictors that could affect surgical success.

\section{Surgical technique}

Written informed consent was obtained from all the patients. A retrograde pyelography was performed in all patients to control the entire renal collecting system. A 0.035 -inch safety guidewire was placed.

In group 1 (sURS group), a second hydrophilic guidewire was carried out into the ureteral orifice through the sURS's (8f or 9f Fr, Karl Storz, Rietheim-Weilheim, Germany) working channel. A semirigid ureteroscope was gently passaged between these two guidewires ("railroad" technique) [10]. The optical ureteral dilatation was done with sURS, and the entire ureter was assessed for anatomy, additional pathologies, and calibration of the ureter. Then, a UAS of an appropriate diameter was placed just below the ureteropelvic junction for renal stones and just below the stone for upper ureteral stones under fluoroscopic guidance.

In group 2 (non-sURS group), the UAS was inserted directly by gliding over the working guidewire. First, a 10-12 f or a 11-13 f UAS was tried. If these sizes were unable to pass to the collecting system, or there was stenosis in the ureter during sURS (for group 1), a smaller UAS was tried under fluoroscopic control. If all attempts failed, insertion of a bare flexible URS (fURS) was tried over guidewire. If this attempt was unsuccessful, the procedure was stopped and a JJ stent was placed and the patient was scheduled for reoperation after three or four weeks. 
After the UAS was placed, renal stones were fragmented by a holmium:YAG laser. Laser energy and pulse frequency were varied based on stone burden and density. If possible, lower pole stones were repositioned into the upper or middle calyx. Stone fragments over 2-3 $\mathrm{mm}$ were extracted by a nitinol basket catheter. A JJ stent was usually left in place according to surgeon preference.

During follow-up, the urinary ultrasound and KUB radiography were done in the follow-up visit after the first month. NCCT was performed in suspicious and necessary cases. Stone-free status was defined as no residual fragments or the presence of residual fragments up to $3 \mathrm{~mm}$. Surgical success was defined as patients' achievement of stone-free status after a single lithotripsy session without the need for additional sessions or ancillary procedures.

\section{Statistical analysis}

All statistical tests were performed using SPSS Statistics version 24 (IBM, Armonk, NY, USA) software. The sample mean was used to determine the average of collected data as quantitative variables met the normal distribution; otherwise, the sample median was used. A chi-square test was performed for nominal variables in the groups. A Student's t-test was applied to make group comparison when the normality assumption was satisfied for both groups. If the normality assumptions were not satisfied for either group or both groups, the equivalent nonparametric Mann-Whitney U test was applied.

Binomial logistic regression was then performed analysing the statistically significant univariate factors. Predictors that obtained significance for surgical success were entered into a multivariable logistic regression model to determine the independent predictors.

\section{RESULTS}

The study consisted of 422 consecutive patients (113 in Group 1; 289 in Group 2) that meet the criteria for inclusion. Of these, $179(42 \%)$ were females and $243(58 \%)$ were males, with a mean age of $48.8 \pm 14$ (14-80) years. Patients' demographics, baseline stone status and characteristics and operative outcomes are presented in Table 1.

Group 1 (sURS group) and group 2 (non-sURS group) patients' comparisons are also listed in Table 2. There was no statistically significant difference between the two groups in terms of stone and patient characteristics. However, the mean operation time was significantly longer in the sURS group $(87.1 \pm 37 \mathrm{~min}$ vs $67.3 \pm 27$ $\min , p<0.0001)$. Fluoroscopy times were similar between the groups $(p=0.53)$. UAS was unable to be placed in four $(3.0 \%)$ patients in the sURS group and $25(8.7 \%)$ patients in non-sURS group $(p=0.03)$. In the non-sURS group a fURS was unable to be inserted through a working guidewire in two patients whose UASs were unable to be placed as well. The JJ stent was placed and reoperation was planned in these patients. While the surgical success was $94.7 \%$ in the sURS group, it was $84.1 \%$ in the non-sURS group, and this was statistically significant $(p=0.002)$. The intraoperative complication rate was lower in the sURS group than in the non-sURS group (1 (0.8\%) vs $14(4.8 \%), p=0.04)$. In the sURS group, the stone could not be properly visualized in one $(0.7 \%)$ patient due to intraoperative bleeding. The complications observed in the non-sURS group included eight $(2 \%)$ mucosal injuries requiring stent insertion, two $(0.6 \%)$ postoperative prolonged haematuria, two $(0.6 \%)$ collecting system perforations requiring JJ stent placement, one $(0.3 \%)$ inability to reach stone and one $(0.3 \%)$ converted to percutaneous nephrolitomy in the same session. Although postoperative complications were lower in the sURS group compared to the non-sURS group, this was not statistically significant $(5(3.8 \%)$ vs $23(8.0 \%), p=0.10$, respectively). The postoperative complications for the sURS group were: fever requiring antibiotic $(\mathrm{n}=2,1.5 \%)$, renal colic requiring analgesic $(\mathrm{n}=2,1.5 \%)$, gross haematuria not requiring transfusion $(\mathrm{n}=1,0.7 \%)$; for the non-sURS group: fever requiring antibiotic $(\mathrm{n}=7,2.4 \%)$, renal colic requiring analgesic $(\mathrm{n}=10,3.4 \%)$, gross haematuria not requiring transfusion $(\mathrm{n}=5$, $1.7 \%)$ and urosepsis $(\mathrm{n}=1,0.3 \%)$.

The results of univariate analysis of the factors affecting surgical success are presented in Table 3. Although the surgical success was determined to be significantly affected by the optical dilatation through sURS, it was not significant in multivariate analysis (in univariate analysis: $p=0.002$, in multivariate analysis: $p=0.179$ 
). We have found two independent factors predicting surgical success in multivariate analysis. These were stone number $(p<0.0001$, odds ratio: 2.28 and $95 \%$ CI [1.48-3.49]) and failed UAS placement $(p=0.035$, odds ratio: 3.49 and $95 \%$ CI [1.04- 11.14]) (Hosmer-Lemeshow test: $p=0.378$ ).

\section{DISCUSSION}

This study is the first research investigating the impact of optical dilatation with sURS on the operative outcomes of RIRS. Our study has revealed two important issues regarding the use of sURS in patients undergoing RIRS. First, when using sURS, UAS is more easily placed into the ureter, and the surgical success rate increases. However, it is not an independent predictor of surgical success. Second, intraoperative complication rates are found to be low.

One of the most essential components of RIRS surgery is the placement of UAS. The use of UAS makes a positive contribution to the operative visibility, stone-free rate, and operation time without increasing the complication rates $[1,3,4,11]$. Furthermore, it is stated in several reports that the utilization of a UAS may have a positive impact on complication rates [8,12]. However, the insertion of a UAS can sometimes be challenging. The authors recommend various strategies to address this problem. Passive ureteral dilatation made with routine stent placement can be safe and efficient, but this method carries the risks of secondary anaesthesia, an operation, and eventually higher costs [1,11]. In addition, stent-related symptoms may be seen, such as prolonged haematuria, flank pain, dysuria and urgency, and patients are usually not willing to accept presenting when they hear about these side-effects [4]. Placement of UAS following active dilatation with a balloon or a coaxial dilator is not routinely recommended because of the risk of significant ureteral injury $[1,5]$. In the EAU guidelines for approximately 10 years it has been advised that sURS before RIRS can be helpful for optical dilatation. The direct visualization of the whole ureter by semirigid ureteroscopy just before the UAS placement is not only to provide optical dilatation but also allow to evaluation of any additional stones, strictures, or tumours in the ureter [11]. Moreover, it can also help with evaluation of ureteral compliance and diameter [5].

Due to a difficult impassable ureter, the failure rates of primary access of UAS range from $6 \%$ to $22 \%$ in the literature $[1,5,13]$. Success rates increase when the appropriate diameter is determined with sURS and when a thinner UAS is used in cases where it is needed [5]. However, the preferred UAS must be narrow enough not to damage the ureter, but wide enough to clean the stone and provide intrarenal circulation. The most reliable method to determine appropriate UAS diameter is to evaluate with sURS. Lima et al. recommend a routine sURS for passive ureteric dilatation and selection of the correct UAS size [8]. In our study, in accordance with the literature the UAS failure rates are $8.7 \%$ in the non-sURS group and $3.0 \%$ in the sURS group. Diameters of sURS used in the study are 8 or $9.5 \mathrm{fr}$ in circumference at the distal tip and $12 \mathrm{fr}$ in circumference at the proximal tip. We think that the mechanism of cascading diameter increase dilates the intramural ureter, the narrowest and least elastic part of the ureter, without damage shown in Figure 1.

Despite all these advantages of UAS, there are some drawbacks. One of these is deterioration of ureteral blood flow. Lallas et al. show transient decreased ureteral blood flow secondary to the use of a UAS in animal models [14]. However, they stated that the compensatory mechanisms of the ureteral wall restored the blood flow of the ureter wall and the integrity of the ureter was preserved. The authors concluded that the use of a UAS with RIRS might be safe; however, care must be taken in selecting an appropriate size of sheath and the duration of surgery should not be prolonged because of the risk of stricture development. In our study, the duration of the operation was found to be significantly higher in the sURS group than in the non-sURS group (the difference between means: $20 \mathrm{~min}, p<0.0001$ ). The extended duration of the operation is thought to have been spent on sURS, but unfortunately the duration of UAS placement has not been measured.

Another drawback associated with UAS is the risk of ureteral injury during entry. Traxer and Thomas prospectively evaluated ureteral injuries secondary to insertion of a 14F UAS [15]. The authors reported that ureteral wall injuries occurred in $46.5 \%$ of the patients, and that the most significant predictor of severe ureteral injury was the absence of stenting before RIRS. However, in another prospective study on 2239 patients treated with fURS, Traxer et al. found that UAS usage did not increase the risk of ureteral wall 
damage, and postoperative infectious complications were reduced [16]. In a retrospective study in which 4500 RIRS procedures were evaluated, intraoperative incidents occurred during $5.2 \%$ of the procedures, and overall complications occurred in $18.9 \%$ [12]. The authors reported that in $4.8 \%$ of the cases in which a ureteral access sheath was used they encountered grade 2 and 3 ureteral wall lesions. In our study, while one $(0.7 \%)$ intraoperative complication (inability to reach stone) was observed in the sURS group, $14(4.8 \%)$ intraoperative complications were observed in the non-sURS groups and the majority of these complications were mucosal injury (57\%). We suppose that optical dilatation with sURS and selection of the correct UAS size reduce the intraoperative complications. Although the rates of postoperative complications in the sURS group were lower, there was no statistical difference between sURS and non-sURS groups.

In many studies, factors that predict stone-free and surgical success have been investigated. In some studies stone size, presence of lower pole calculi, surgical experience, presence of hydronephrosis, and UAS use are significant predictors of RIRS outcome, while others have only found the number of stones and the number of sites [17-19]. Especially thanks to the advances in the field of lasers, stone access is the most important factor that makes treatment possible. In our series, the mobile lower pole stones were repositioned into the upper or middle calyx. This method may have reduced the impact of localization on the stone-free rate. In our study, UAS usage and the presence of multiple stones were found as independent predictors for surgical success. The failed surgery rates have increased 2.28 times as the number of stones increases, and have increased 3.49 times when the UAS cannot be placed. It is expected that the use of sURS before RIRS increases surgical success by increasing the rate of UAS placement and reducing the rate of intraoperative complications. Finally, we found that the surgical success rate was higher in the sURS group $(\mathrm{p}=0.002)$. However, our study found that sURS had no independent effects on the surgical success of RIRS.

\section{CONCLUSION}

As a result, the optical dilatation with sURS, which is also recommended in EAU guidelines, makes a positive contribution to surgical success, facilitating UAS placement and leading to a low complication rate. Although it requires longer surgery time, we recommend this method, with acceptable fluoroscopy time, in patients with no previous history of passive dilatation using a JJ stent. However, further randomized prospective studies are needed.

\section{Limitations}

Our study is retrospective in nature, which may lead to selection bias. We tried to overcome this limitation by including all cases operated for kidney and upper ureteral stones during the study period. The data of four different centres were included in the study. While the multicentric nature of the study increases the quality, doubts about the technique arise from different surgeons operating. However, the surgeons in different centres have at least five years of experience and the surgical steps used are similar. Removal of the use of balloons and coaxial dilators from the study provides method harmony and shows the direct effect of our technique. Other conditions, such as previous same-side ureteroscopy or JJ stent and known renal anatomical abnormalities that may overshadow the effectiveness of sURS for optical ureteral dilatation and selection of the correct UAS size, have been excluded from the study. Other drawbacks of the study are that while operation and fluoroscopy times are calculated, the duration of UAS has not been calculated, and the lack of long-term follow-ups and stenosis rates.

\section{Acknowledegements: None}

Conflicts of interest: The authors have declared that they have no conflicts of interest.

\section{Funding: None}

\section{REFERENCES}

1. Mogilevkin Y, Sofer M, Margel D, Greenstein A, Lifshitz D. Predicting an effective ureteral access sheath insertion: a bicenter prospective study. J Endourol. 2014;28(12):1414-7. 
2. Karsiyakali N, Karabay E, Erkan E, Kadihasanoglu M. Evaluation of Nephrolithometric Scoring Systems to Predict Outcomes of Retrograde Intrarenal Surgery. Urol J. 2020;4(10).

3. Shvero A, Herzberg H, Zilberman D, Mor Y, Winkler H, Kleinmann N. Is it safe to use a ureteral access sheath in an unstented ureter? BMC Urol. 2019;19(1):019-0509.

4. Lee MH, Lee IJ, Kim TJ, Lee SC, Jeong CW, Hong SK, et al. The effect of short-term preoperative ureteral stenting on the outcomes of retrograde intrarenal surgery for renal stones. World J Urol. 2019;37(7):1435-40.

5. Boulalas I, De Dominicis M, Defidio L. Semirigid ureteroscopy prior retrograde intrarenal surgery (RIRS) helps to select the right ureteral access sheath. Arch Ital Urol Androl. 2018;90(1):20-4.

6. Türk C, Knoll T, Petrik A, Sarica K, Straub M, Seitz C. Guidelines on urolithiasis. European association of urology. 2011.

7. Türk C, Neisius A, Petik A, Seitz C, Skolarikos A, Thomas K. Guidelines on urolithiasis. European Association of Urology. 2020.

8. Lima A, Reeves T, Geraghty R, Pietropaolo A, Whitehurst L, Somani BK. Impact of ureteral access sheath on renal stone treatment: prospective comparative non-randomised outcomes over a 7-year period. World J Urol. 2020;38(5):1329-33.

9. Perks AE, Schuler TD, Lee J, Ghiculete D, Chung DG, RJ Dah, et al. Stone attenuation and skin-tostone distance on computed tomography predicts for stone fragmentation by shock wave lithotripsy. Urology. 2008;72(4):765-9.

10. Duty B, Conlin M. Principles of urologic endoscopy. Campbell-Walsh Urology 11th ed Philadelphia, PA: Elsevier. 2016.

11. Karabulut I, Keskin E, Bedir F, Yilmazel FK, Ziypak T, Doluoglu OG, et al. Rigid Ureteroscope Aided Insertion of Ureteral Access Sheath in Retrograde Intrarenal Surgery. Urology. 2016;91:222-5.

12. Geavlete PA, Mulţescu R, Geavlete BF. Morbidity of Retrograde Flexible Ureteral Approach for PyeloCaliceal Stones - A Retrospective Review of 4500 Procedures. Chirurgia. 2020;115(1):63-8.

13. Cho SY, Ryang SH, Lee DS. A presumptive role of lower ureteral angles in the difficulty of ureteral access sheath insertion during retrograde intrarenal surgery. Int Urol Nephrol. 2020;29(10):020-02483.

14. Lallas CD, Auge BK, Raj GV, Santa-Cruz R, Madden JF, Preminger GM. Laser Doppler flowmetric determination of ureteral blood flow after ureteral access sheath placement. J Endourol. 2002;16(8):583-90.

15. Traxer O, Thomas A. Prospective evaluation and classification of ureteral wall injuries resulting from insertion of a ureteral access sheath during retrograde intrarenal surgery. J Urol. 2013;189(2):580-4.

16. Traxer O, Wendt-Nordahl G, Sodha H, Rassweiler J, Meretyk S, Tefekli A, et al. Differences in renal stone treatment and outcomes for patients treated either with or without the support of a ureteral access sheath: The Clinical Research Office of the Endourological Society Ureteroscopy Global Study. World J Urol. 2015;33(12):2137-44.

17. Ito H, Sakamaki K, Kawahara T, Terao H, Yasuda K, Kuroda S, et al. Development and internal validation of a nomogram for predicting stone-free status after flexible ureteroscopy for renal stones. BJU Int. 2015;115(3):446-51.

18. Tonyalı Ş, Yılmaz M, Karaaslan M, Ceylan C, Işıkay L. Prediction of stone-free status after single-session retrograde intrarenal surgery for renal stones. Turk J Urol. 2018;44(6):473-7.

19. Cho SY, Choo MS, Jung JH, Jeong CW, Oh S, Lee SB, et al. Cumulative sum analysis for experiences of a single-session retrograde intrarenal stone surgery and analysis of predictors for stone-free status. PLoS One. 2014;9(1). 


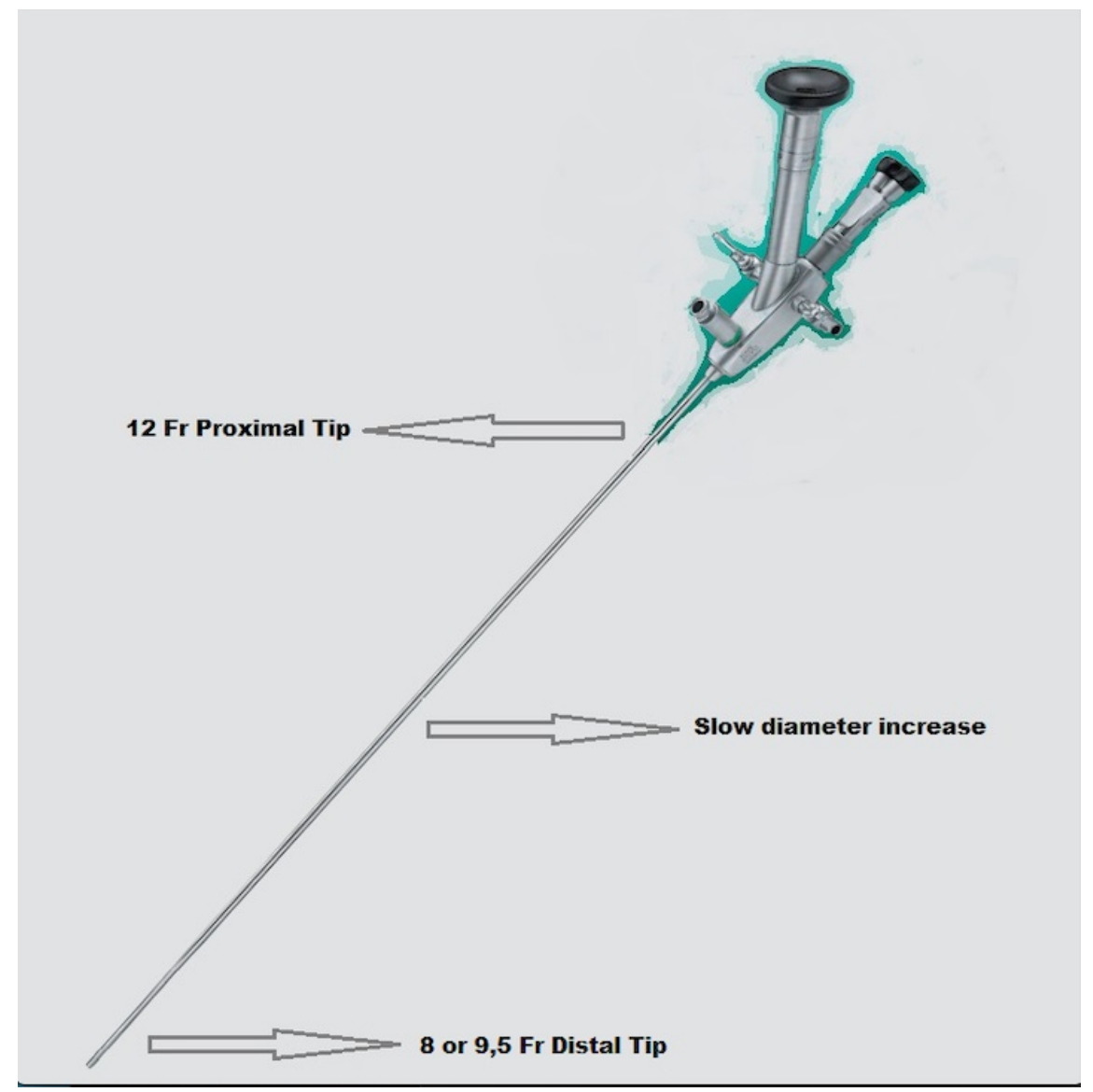

\section{Hosted file}

Table 1.pdf available at https://authorea.com/users/375410/articles/516584-the-effect-ofoptical-dilatation-before-retrograde-intrarenal-surgery-on-success-and-complicationsresults-of-the-rirsearch-group-study

\section{Hosted file}

Table 2.pdf available at https://authorea.com/users/375410/articles/516584-the-effect-ofoptical-dilatation-before-retrograde-intrarenal-surgery-on-success-and-complicationsresults-of-the-rirsearch-group-study

\section{Hosted file}

Table 3.pdf available at https://authorea.com/users/375410/articles/516584-the-effect-ofoptical-dilatation-before-retrograde-intrarenal-surgery-on-success-and-complicationsresults-of-the-rirsearch-group-study 